\title{
Theory of Nonsinusoidal Small Antennas with Applications to Near-Field Communication System Analysis and Design
}

\author{
Said Mikki*
}

\begin{abstract}
We provide a conceptual and theoretical analysis of nonsinusoidal antennas with emphasis on how electromagnetics and communication theories can be integrated to propose ideas for near-field (NF) communications systems utilizing future antennas. It is shown through rigours analysis that in nonsinusoidal antennas it is possible to derive and solve ordinary differential equations giving specialized time-domain excitation signals that lead to exact cancellation of the near field at specific radiation spheres. This opens the door to building NF communications systems with far-field-like communication receiver infrastructures utilized if the receive antenna is placed at the special sphere where the $\mathrm{NF}$ component is made to vanish. We deploy exact current Green's function analysis method and completely avoid the use of any frequency-domain method. Complete expressions of the electromagnetic near- and far- field contributions to all signals propagating from the source to the receiver are then derived and their physical content discussed. The distortion effects and signal-to-noise rations due to the near-field are also identified and derived theoretically. It is found that using this specialized pulse excitation method in nonsinusoidal antennas, distortion caused by near-field components can be eliminated at critical distances between the source and the receiver. Realization issues of this system are briefly discussed together with some potential applications.
\end{abstract}

\section{INTRODUCTION}

We define a nonsinusoidal antenna (NSA) as a carrier-free structure emitting electromagnetic energy by directly modulating the radiating current distribution over the antenna surface through specialized current sources (drivers) connected the antenna input terminals. The single most important character of these systems is that they are not conceptually and theoretically based on the powerful and extremely popular analytical paradigm called frequency-domain analysis. Historically speaking, the period following the end of Second World War was characterized by a remarkably persistent emphasis on frequency-domain methods in spite of the fact that time-domain techniques were widely considered in the first half of the twentieth century side in side with harmonic analysis techniques. Sinusoidal antennas are radiating structures either excited by a single-tone signal or by (most likely in today's wireless applications) modulated signals with high-frequency sinusoidal carrier. When the relative bandwith of the modulated signal is small (narrowband electromagnetic waves), the most dominant situation encountered in applications to date, engineers often ignore the difference between a pure sinusoidal signal and a modulated digital pulse. However, the two cases remain fundamentally different. A pure sinusoidal antenna is inherently noncausal since the switching on time of the sinusoid is shifted to the infinite past, raising potential problems when the antenna is utilized in digital communication links with relatively large bandwith. On the other hand, a switched or pulsed carrier (modulated signal) is essentially causal. Only when the baseband signal bandwidth $B$ is very small compared with the carrier frequency $f_{c}$, i.e., when the condition

$$
B \ll f_{c}
$$

* The author can be reached at said.m.mikki@gmail.com. 
is satisfied, one may say that the two cases - the noncausal sinusoidal antenna and the causal pulsed sinusoid - are quantitatively the same.

The situation has began to change in recent years with the rise of Ultra-Wideband (UWB) antennas, where the emphasis shifted from modulated signals with relatively small fractional bandwidth to signals with very wide spectral span [1]. However, that does not imply that the theory of nonsinusoidal antennas is identical to UWB antennas. It seems that most research on UWB systems still utilizes Fourier transform techniques, which is obvious from the fact that the very name of the system itself, ultrawide-band, implies frequency-domain concepts like bandwidth. Moreover, research strategies in UWB antenna system analysis and design continue to think of the antenna as essentially a frequency-domain device with "flattened" or extended operational spectral band where multiple single-frequency antenna responses are stacked and concatenated next to each other in order to produce a total wide band (preferably flat) frequency response extended over a very large bandwidth. NSAs appear to deviate from this trend as their theories continue to intentionally and consistently avoid working in the frequency domain altogether.

Fundamental criticism of the unquestioned use of Fourier transforms to infer conclusions in the time domain from the frequency domain is not new, but was delivered in a sustained and powerful form throughout the work of Harmuth, most notably [2], who wrote extensively on nonsinusoidal electromagnetic systems (waveguides, radars, sensors, antennas). For example, analytical formulas for nonsinusoidal travelling wave antennas were derived in [3]. A modified radar equation for nonsinusoidal waves was formulated in [4]. A specialized type of electrically-small nonsinusoidal antennas, e.g., the Large-Current Radiator (LCR), was proposed in [5], [6], [7] as a wideband carrier-free antenna alternative to traditional sinusoidal resonant small antennas. LCRs were further developed in [8], [9], [10].

Since signals (waveforms capable of carrying new information) must be nonanalytic functions, sinusoidal and periodic functions (standard examples of analytic functions) cannot carry information. Therefore, a correct and rigorous unified treatment of electromagnetic communication systems as information-processing systems requires solving Maxwell's equations when the forcing term assumes a basic nonanalytic shape like step functions. Solutions obtained early by Stratton using the Laplace transform in the 1940s were shown to be not the only ones possible as alternative solutions using different methods produced nonidentical answers [2]. This has lead to some controversy, especially given the proposal by Harmuth to modify Maxwell's equations by including magnetic current terms that can be removed in the end by a limiting process [11], [12]. We note that for transient problems where the excitation is applied at a finite time $t=t_{0}$, the Laplace transform is often preferred to the Fourier transform. But given the well known mathematical fact that the inverse Laplace transform is not unique, as pointed out correctly in connection with the Harmuth proposal in [13], the use of frequencydomain methods becomes limited in the very general case of arbitrary time-domain (nonanalytic) signal transmission through electromagnetic infrastructure. Such criticism of the unrestricted deployment of Fourier transform methods for solving electromagnetic wave propagation problems has lead to the revival of the controversy about how best to define signal propagation speed (inadequacy of the group velocity concept [14]). The climax, however, was the proposed correction of Maxwell's equations given in [11], [12] and further elaborated in [15]. ${ }^{\dagger}$ Other writers have also voiced similar skepticism regarding the unhinged use of frequency-domain methods to infer data and information about the time-domain behaviour of electromagnetic waves, e.g., see the recent book [16] and the papers [17-20].

The recent interest in electromagnetic near fields has pushed researchers to investigate alternative theoretical [21-23, 23, 24], experimental [21, 25, 26], and computational [21,27-31] tools capable of realizing and demonstrating new dimensions and devices in engineering electromagnetics, and hence the motivation behind this work aiming at reexamining the usual views on antennas by exploiting the subtle but often neglected relation between space and time in radiation problems. In this paper, we follow Harmuth's suggestion to work with nonsinusoidal antennas completely in the time domain, avoiding as much as possible using frequency-domain concepts. However, at the same time we will not adopt in the present work Harmuth's latter proposal to modify Maxwell's equations. Instead, we continue to work with the standard set of Maxwell's equations though expressed in space-time rather than frequency-space. The main objectives of this work are the following. First, we would like to present

\footnotetext{
$\dagger$ In this paper, the controversial subject of Harmuth modification of Maxwell's equation will not be taken up. All electromagnetic
} derivations presented below will be based on the classical Maxwell's equations. 
an illuminating example of how electromagnetic and communication theories can be merged together in a single formalism, that of time-domain (nonsinusoidal) antennas where the purely communication theoretic aspects of the problem, such as the design of the digital receiver, are developed with an eye on the electromagnetic infrastructure of the wireless link. Second, we demonstrate this in a particular case of Near-Field Communication (NFC) system where the receiving antenna is brought close to the transmitting antenna near-field zone. We will show that it is possible to engineer the near-field structure of the link by harnessing the full power of nonsinusoidal radiators as antennas with fully arbitrary timedomain waveforms. This will lead to a specialized system where the near field can be forced by the designer to completely vanish at a particular sphere (the causal sphere), the location where we intend to place the receiving antenna. It turns out that the design of the digital receiver can be considerably simplified and the distortion produced by near-field terms totally eliminated if we excite the Tx NSA with a specialized decaying pulse that can be derived by solving an exact differential equation to be derived below. If the digital link utilizes this special decaying pulse as the digital transmission main pulse, then the resulting NFC system utilizing NSAs can be made distortion-free and only far-field forms will be seen by the receiving antenna even at positions deep into the near-zone region of the Tx NSA. This remarkable result turns out to be possible only when no sinusoidal carrier is used and hence a unique feature of NSAs compared with traditional resonant antennas like Hertzian dipoles.

One of the main objectives of the present work is exploiting the NSA as a good illustrative example demonstrating the power and importance of pursuing a unified approach to electromagnetics and communications in the analysis and design of wireless networks. Although this paper is essentially conceptual and intentionally developed at the very general level, it is interested in showing that fundamental physical considerations established based on general sound mathematical principles, e.g., Green's functions, can lead to considerable insight into how real-life communication systems work. By focusing on a specific generic case, the near-field communication (NFC) link, we also provide a more narrowed down version of our general conclusions regarding the importance to analyzing communication systems in the context of an exact electromagnetic spacetime system theory [32], not traditional signal processing. We stress here that no final realization or physical device implementation is given here. The experimental and numerical investigation of nonsinusoidal antennas has already been reported elsewhere; in fact, it has been under continuous investigation since the 1970s. Some literature survey of he subject and further remarks and proposals about applications and realization are collected in Sec. 4. Readers interested in the device level aspect can consult some of the literature cited there. However, since the present paper is interested in providing a general conceptual framework for several new ideas at the intersection of electromagnetic and communication theories, we have opted for working with exactly solvable nonsinusoidal antenna model, where Maxwell's equations are solved for the electromagnetic fields radiated by a small antenna. All results obtained below in Sec. 2 and 3 are exact. Departure from the exact theory are provisionally discussed in Sec. 4 where the need for numerical optimization and possibly the use of full-wave simulation is anticipated in conjunction of future work on the subject.

\section{NONSINUSOIDAL ANTENNA-BASED NEAR-FIELD COMMUNICATION LINK ANALYSIS AND DESIGN}

\subsection{Antenna Transmitting Mode Analysis}

Consider an electric dipole antenna with length $l$ oriented along the $\hat{l}$ direction. There exists timedependent charges $q(t)$ and $-q(t)$ located at the two ends of the dipole, which is further assumed to be short such that these charges do not depend on the position on each arm. In this way, an idealized electric dipole can be realized as short thin-wire antenna (thin strip, thin cylinder, etc). The dipole moment $\mathbf{p}(t)$ is defined by [33]

$$
\mathbf{p}(t)=q(t) \hat{l},
$$

and is related to the current $i(t)$ through

$$
\frac{\mathrm{d} \mathbf{p}(t)}{\mathrm{d} t}=\mathbf{i}(t)=i(t) \hat{l} .
$$


Since the dipole is assumed to be small, the current distribution is also independent of position, and the quantity $i(t)$ measured in A represents an average over the small sectional area of the dipole. The current will radiate electric and magnetic fields $\mathbf{E}(\mathbf{r}, t)$ and $\mathbf{H}(\mathbf{r}, t)$ throughout the exterior region $V_{\mathrm{ex}}$ of the antenna. These can be computed in terms of the vector and scalar potentials $\mathbf{A}(\mathbf{r}, t)$ and $\varphi(\mathbf{r}, t)$ by means of the following Lorentz-gauge formulas [34], [35]

$$
\mathbf{E}(\mathbf{r}, t)=-\frac{\partial \mathbf{A}(\mathbf{r}, t)}{\partial t}-\nabla \varphi(\mathbf{r}, t), \quad \mathbf{H}(\mathbf{r}, t)=\left(c / Z_{0}\right) \nabla \times \mathbf{A}(\mathbf{r}, t),
$$

where $c=1 / \sqrt{\mu_{0} \epsilon_{0}}$ is the vacuum speed of light and $Z_{0}:=\mu_{0} / \epsilon_{0}$ is the vacuum wave impedance. ${ }^{\ddagger}$

Calculations of the scalar and vector potentials can be considerably simplified if we use the assumptions

$$
l / r \ll 1, \quad \frac{\partial}{\partial \mathbf{r}} i(\mathbf{r}, t)=0 .
$$

Here, $r$ refers to a generic near- or far-field observation position. The relation $l / r \ll 1$ means that we observe the field only at locations sufficiently far away from the small dipole such that the ratio $l / r$ can be considered very small. The physical smallness of the dipole allows us to bring $r$ close to the antenna while still satisfying this condition. The exterior region $V_{\mathrm{ex}}$ is defined as the region surrounding the NSA where (5) is satisfied. Moreover, we further assume that the current is uniform along the antenna to mimic the situation with infinitesimal dipoles. This considerably simplifies the analysis to follow although it can be relaxed in future work. ${ }^{\S}$ The conditions (5) were designed to capture for NSAs what is usually considered "electrically small antennas" (ESA) in the frequency-domain literature. We observe that for nonsinusoidal antennas, it is not possible to directly apply the frequency-domain concepts familiar in conventional antenna theory. In particular, the traditional definition of small antennas as electrically small antennas satisfying $d / \lambda \ll 1$ cannot be invoked in our case since there is no clear definition of wavelength in arbitrary time-varying (nonsinusoidal) antennas. For that reason, we will deploy the condition (5) as our main definition of small antennas in this paper." From the conditions (5) it can be shown that [7], [34]

$$
\mathbf{A}(\mathbf{r}, t)=\frac{Z_{0} i(t-r / c)}{4 \pi c r} \mathbf{l}
$$

where $\mathbf{l}=l \hat{l}$. Here, as in every other expression involving the current $i$ or charge $q$, the retarded time $t_{r}$

$$
t_{r}:=t-r / c
$$

is inserted to account for causality. The scalar potential can also be computed and is readily found to be

$$
\varphi(\mathbf{r}, t)=\frac{Z_{0} c}{4 \pi}\left(\frac{1}{r^{3}} \int \mathrm{d} t_{r} i\left(t_{r}\right)+\frac{1}{c r^{2}} i\left(t_{r}\right)\right) \mathbf{l} \cdot \mathbf{r} .
$$

We reemphasize here that both (6) and (8) are approximation based on neglecting terms of order $O(l / r)^{2}$ and higher. ${ }^{\top}$ From these expressions for the electromagnetic potentials $\mathbf{A}$ and $\varphi$, the electric and magnetic fields can be computed by means of (6) and (8) to yield

$$
\begin{gathered}
\mathbf{E}(\mathbf{r}, t)=\frac{Z_{0} l}{4 \pi c}\left\{\frac{1}{r} \frac{\mathrm{d} i\left(t_{r}\right)}{\mathrm{d} t} \hat{r} \times \hat{r} \times \hat{l}+\left(\frac{c}{r^{2}} i\left(t_{r}\right)+\frac{c^{2}}{r^{3}} \int \mathrm{d} t_{r} i(t r)\right)[\hat{r} \times \hat{r} \times \hat{l}+2(\hat{l} \cdot \hat{r}) \hat{r}]\right\}, \\
\mathbf{H}(\mathbf{r}, t)=\frac{l}{4 \pi c}\left(\frac{1}{r} \frac{\mathrm{d} i\left(t_{r}\right)}{\mathrm{d} t}+\frac{c}{r^{2}} i\left(t_{r}\right)\right) \hat{l} \times \hat{r} .
\end{gathered}
$$

$¥$ As usual, $\mu_{0}$ and $\epsilon_{0}$ stand for the standard magnetic permeability and electric permitivity of free space, respectively.

$\S$ In practice, very short wires or longer wires with end loadings can realize such uniform current required in our definition of small NSA given by (5).

\| Similarly, as we will see later, the definition of the near field will not use the concept of the center wavelength.

I In fact, terms of order $O(l / r)$ already cancel out and do not contribute to $\mathbf{A}$, see [7]. 
The terms involving $(1 / r) \mathrm{d} t / \mathrm{d} t$ in (9) and (10) are the familiar far-field components of the electric dipole radiation fields, which have been studied extensively in electrodynamics and antenna engineering [33], [36]. On the other hand, the electric field also involves additional terms proportional to $1 / r^{2}$ and $1 / r^{3}$, which are responsible for the near-field part. For the magnetic field, however, only an $1 / r^{2}$-term is involved. In traditional sinusoidal antenna theory, the near field zone is delimited by measuring the electric distance (normalized distance with respect to the center frequency wavelength). In nonsinusoidal antennas, this is not straightforward because we would like to avoid the use of any specific center frequency in the antenna analysis and design. Therefore, in this paper, we define the near field of the antenna as the part containing all terms involving radial dependence of the order $1 / r^{2}$ and higher. ${ }^{+}$The terms containing $\hat{r} \times \hat{r} \times \hat{l}$ in (9) are transverse field components, while those with $(\hat{l} \cdot \hat{r}) \hat{r}$ are longitudinal fields. However, the magnetic field in (10) appears to contain only transverse components. ${ }^{*}$ Furthermore, we note that the time dependence of the electromagnetic fields is very different in the near- and far-field terms. For both the electric and magnetic fields, the far-field terms are proportional to the first-order time derivative of the retarded current, a very well known result. On the other hand, near-field components, i.e., all terms involving $1 / r^{2}$ and $1 / r^{3}$, have essentially different functional time variations. This observation is crucial for near-field communications. In fact, it is clear that because of this nonuniformity in the temporal contents of the near- and far-field terms, distortion is bound to happen in near-field communication systems unless one tries to avoid receiving the near-field part. In Sec. 3, we impose a specialized temporal dependence on the driving current $i(t)$ in order to completely cancel the near-field components of the electric field, which is expected to reduce the complexity of the NFC receiver by eliminating distortion. ${ }^{\sharp}$

\subsection{Antenna Receiving Mode Analysis}

In the receiver part of the system, we need to estimate the $\mathrm{Rx}$ signal based on the impinging field generated above. For simplicity, in this work we assume only perfectly electric conducting (PEC) antennas, which implies that only the impinging electric field is relevant for the determination of the Rx signal (the magnetic field does not interact with PEC antennas.) The Rx antenna has a surface $S_{\mathrm{rx}}$ and is completely described by its spacetime antenna current Green's function (ACGF) $\overline{\mathbf{F}}_{\mathrm{rx}}\left(\mathbf{r}_{p}, \mathbf{r}^{\prime} ; t-t^{\prime}\right)$, with $\mathbf{r}_{p}$ the $\mathrm{Rx}$ port location at which the $\mathrm{Rx}$ current signal $\mathbf{J}\left(\mathbf{r}_{p}, t\right)$ will be collected. It has been recently shown that $[32]$

$$
\mathbf{J}\left(\mathbf{r}_{p}, t\right)=\int_{S_{\mathrm{rx}}} \int_{-\infty}^{t-\left|\mathbf{r}_{p}-\mathbf{r}^{\prime}\right| / c} \mathrm{~d} s^{\prime} \mathrm{d} t^{\prime} \overline{\mathbf{F}}_{\mathrm{rx}}\left(\mathbf{r}_{p}, \mathbf{r}^{\prime} ; t-t^{\prime}\right) \cdot \mathbf{E}_{\mathrm{in}}\left(\mathbf{r}^{\prime}, t^{\prime}\right) .
$$

Here, $\mathbf{E}_{\text {in }}$ is the incident field, which will be taken as that computed by (9). The Antenna Current Green's Function (ACGF) $\overline{\mathbf{F}}_{\mathrm{rx}}\left(\mathbf{r}, \mathbf{r}^{\prime} ; t-t^{\prime}\right)$ was introduced in the frequency domain, then expanded into the time domain for wireless communication applications in [32,39-46]. It is a 2-dimensional tensor (dyad) defined on the antenna surface $S_{\mathrm{rx}}$ and provides an exact expression for the current response valid for arbitrary spacetime illumination fields. The main advantage of using the ACGF $\overline{\mathbf{F}}_{\mathrm{rx}}\left(\mathbf{r}_{p}, \mathbf{r}^{\prime} ; t-t^{\prime}\right)$ is that it provides a signal-processing-like rigorous electromagnetic response function suitable for wireless communications research. Since electromagnetic fields are the main carriers of information and energy but fields are spacetime functions, not signals, the ACGF provides a detailed explication of the internal space-time coupling mechanism needed in order to unlock new potentials of electromagnetic communication systems [46-48]. This Green's function also needs to be obtained only once (via special computation or measurement); afterward, it can be used repeatedly in the convolutiontype integral (11) to estimate the Rx signal without the need to recompute the grid or remesh via full-wave methods like FEM, MoM, FDTD, etc.

\footnotetext{
+ This approach is often used in optical near field theories and nano-optics, e.g., see [37] and [38].

* It is sometimes claimed that the signature of near fields is the presence of such longitudinal components in the near zone. However, in this paper, as above, we don't distinguish transverse and longitudinal components in our near/far field classification. In particular, two $1 / r^{2}$ and $1 / r^{3}$ components in (9) are transverse and these are taken here as essential ingredients in the composition of the near field structure.

\# The harmful impact of near-field terms causing distortion was already anticipated theoretically and experimentally in sensor applications. For example, see [6].
} 
We next restrict our attention to the special but important case when the Rx antenna is a small dipole oriented along the direction $\hat{u}$ and centered at $\mathbf{r}_{p}$. In that case, we can approximate the ACGF by

$$
\overline{\mathbf{F}}_{\mathrm{rx}}\left(\mathbf{r}_{p}, \mathbf{r} ; t-t^{\prime}\right) \simeq \overline{\mathbf{F}}_{\mathrm{rx}}\left(\mathbf{r}_{p}, \mathbf{r}_{p} ; t-t^{\prime}\right), \quad \mathbf{r} \in S_{\mathrm{rx}} .
$$

This approximation should be understood in the sense of generalized function theory [49] since the ACGF is not an ordinary function but a distribution [42], [46]. In a nutshell, (12) says that $\int \mathrm{d} s^{\prime}$ integrals containing the Rx ACGF $\overline{\mathbf{F}}_{\mathrm{rx}}\left(\mathbf{r}_{p}, \mathbf{r} ; t-t^{\prime}\right)$ can be approximated by evaluating the integrand at $\mathbf{r}^{\prime}=\mathbf{r}_{p}$, the location of the center of the small NSA. From (12) and (11), it follows that

$$
\mathbf{J}\left(\mathbf{r}_{p}, t\right)=\int_{t_{0}}^{t} \mathrm{~d} t^{\prime} \overline{\mathbf{F}}_{\mathrm{rx}}\left(\mathbf{r}_{p}, \mathbf{r}_{p} ; t-t^{\prime}\right) \cdot \mathbf{E}_{\mathrm{in}}\left(\mathbf{r}^{\prime}, t^{\prime}\right)
$$

where to enforce causality we assume the electric field is applied at the Rx antenna location $\mathbf{r}_{p}$ exactly at time $t_{0}$. It is interesting to note that the causal component of the Rx ACGF, mainly the upper end of the time integral in (11), vanishes under the small NSA condition (12). However, mathematically speaking, (13) does not necessarily follow from (12) because the space and time integrals in (11) cannot be exchanged due to the causality restriction, i.e., the dependence of the upper end of the time integral on location on the antenna [32]. Still, one would intuitively expect true small NSAs to obey relations like (13) since the Rx probe is very small compared with field dimensions. Consequently, we will define small NSA as those antennas satisfying the two conditions (12) and (13). Again, and similar to the Tx NSA definition encapsulated by (5), the smallness of the NSA devices are assessed with only spacetime concepts, while frequency-domain quantities like wavelength are avoided.

For dipole Rx NSAs, the expression (13) can be significantly simplified. If the Rx small dipole antenna is oriented along the unit vector $\hat{\alpha}$, then the RX ACGF can be expanded as [43]

$$
\overline{\mathbf{F}}_{\mathrm{rx}}\left(\mathbf{r}_{p}, \mathbf{r}_{p} ; t-t^{\prime}\right)=\hat{\alpha} \hat{\alpha} F\left(\mathbf{r}_{p} ; t-t^{\prime}\right),
$$

where the scalar function $F$ gives the electromagnetic responsitivity of the antenna in space and time. The dyadic product $\hat{\alpha} \hat{\alpha}$ accounts for the polarization structure of the field-antenna interaction process, with detailed calculus of use explicated in [46]. Substituting (14) into (13) gives the following expression for the Rx signal in the NSA NFC system

$$
\mathbf{J}\left(\mathbf{r}_{p}, t\right)=\hat{\alpha} \int_{t_{0}}^{t} \mathrm{~d} t^{\prime} F\left(\mathbf{r}_{p} ; t-t^{\prime}\right) E_{\mathrm{in}, \mathrm{t}}\left(\mathbf{r}_{p}, t^{\prime}\right),
$$

where

$$
E_{\text {in }, \mathrm{t}}\left(\mathbf{r}^{\prime}, t^{\prime}\right):=\hat{\alpha} \cdot \mathbf{E}_{\text {in }}\left(\mathbf{r}^{\prime}, t^{\prime}\right)
$$

is the tangential component of the incident electric field. If we take the Fourier transform of (15) the following simple relation is obtained

$$
\mathbf{J}\left(\mathbf{r}_{p} ; \omega\right)=\hat{\alpha} F\left(\mathbf{r}_{p} ; \omega\right) E_{\mathrm{in}, \mathrm{t}}\left(\mathbf{r}_{p} ; \omega\right)
$$

where the frequency-domain functions are the Fourier transforms of the corresponding spacetime functions. The relation (17) is the very well known expression of the current induced on a small dipole in the frequency domain. It is derived here rigorously from the more general causal integral (11).

In order to compute the time-domain received signal, we re-express (15) in terms of the convolution operation $*$

$$
\mathbf{J}\left(\mathbf{r}_{p}, t\right)=\hat{\alpha} F\left(\mathbf{r}_{p} ; t\right) * E_{\text {in }, \mathrm{t}}\left(\mathbf{r}_{p}, t\right) .
$$

Using the radiated field expression (9) in (18), we arrive at

$$
\mathbf{J}\left(\mathbf{r}_{p}, t\right)=\mathbf{J}_{\mathrm{FF}}\left(\mathbf{r}_{p}, t\right)+\mathbf{J}_{\mathrm{NF}}\left(\mathbf{r}_{p}, t\right),
$$

where the far- and near-field contributions to the Rx current are denoted by $\mathbf{J}_{\mathrm{FF}}\left(\mathbf{r}_{p}, t\right)$ and $\mathbf{J}_{\mathrm{NF}}\left(\mathbf{r}_{p}, t\right)$, respectively, and are given by

$$
\mathbf{J}_{\mathrm{FF}}\left(\mathbf{r}_{p}, t\right):=\hat{\alpha} \frac{Z_{0} l}{4 \pi c} \hat{\alpha} \cdot(\hat{r} \times \hat{r} \times \hat{l}) \frac{1}{r} F\left(\mathbf{r}_{p} ; t\right) * \frac{\mathrm{d} i\left(t_{r}\right)}{\mathrm{d} t},
$$




$$
\mathbf{J}_{\mathrm{NF}}\left(\mathbf{r}_{p}, t\right):=\hat{\alpha} \frac{Z_{0} l}{4 \pi c}\{[\hat{r} \times \hat{r} \times \hat{l}+2(\hat{l} \cdot \hat{r}) \hat{r}] \cdot \hat{\alpha}\} F\left(\mathbf{r}_{p} ; t\right) *\left(\frac{c}{r^{2}} i\left(t_{r}\right)+\frac{c^{2}}{r^{3}} \int \mathrm{d} t_{r} i\left(t_{r}\right)\right) .
$$

The relations (19), (20), and (21) provide a complete and exact mathematical representation of the timedomain waveforms captured by a Rx NSA placed at position $\mathbf{r}_{p}$ when the fields produced by the Tx NSA are intercepted. It is clear that in NFC systems, and in contrast to traditional RF communications done in the far field, the Rx current is composed of two parts, one solely due to the far field and is proportional to $1 / r$, while the other is the NF contribution and is scaled by factors proportional to $1 / r^{2}$ and $1 / r^{3}$. These expressions will be further analyzed next in order to develop an efficient and simple digital receiver architecture suitable for NFC links based on electromagnetic theory.

\subsection{Digital Receiver Design}

The most striking feature of the detailed Rx signal expressions derived above is that the FF contribution is simply the convolution of the small dipole antenna impulse response $F\left(\mathbf{r}_{p}, t\right)$ with the first-order time derivative of the transmitted current $i(t) .^{\dagger \dagger}$ This is well known and has been investigated extensively in the antenna community. However, the NF expression (21) is very different from the far-field formula (21). Indeed, we may notice that in (21) the time variation of the NF Rx signal $\mathbf{J}_{\mathrm{NF}}(t)$ does not correspond to any simple function of the transmitted current $i(t)$. Instead, $\mathbf{J}_{\mathrm{NF}}(t)$ possesses a complicated timedomain form composed of sum of terms involving directly the transmitted current $i(t)$ and its time integral. Moreover, the former (direct term) is scaled by $1 / r^{2}$ while the latter (integrated term) is proportional to $1 / r^{3}$. Now, in the far zone, one may just ignore the NF current since only the $1 / r$ survives in the infinite sphere, that is

$$
\lim _{r_{p} \rightarrow \infty} \frac{\left|\mathbf{J}_{\mathrm{NF}}\left(\mathbf{r}_{p}, t\right)\right|}{\left|\mathbf{J}_{\mathrm{FF}}\left(\mathbf{r}_{p}, t\right)\right|}=0
$$

But in the intermediate NF zones of interest in modern NFC systems the situation is altogether different. If the Rx antenna is very close to the Tx, as in nanoscale communications or some NF connectivity applications that are becoming popular nowadays (e.g., device-to-device, peer-to-peer, machine-tomachine, backhaul networks, etc), then the $1 / r^{3}$ terms may dominate the contribution to the Rx signal. In such case, the FF signal involving $\mathrm{d} i / \mathrm{d} t$ will be contaminated by another term involving $\int \mathrm{d} t i(t)$. More problematic is that as the $\mathrm{Rx}$ gets a bit farther from the $\mathrm{Tx}$, then the $1 / r^{2}$-term contribution, which brings $i(t)$ directly into the total Rx current, may dominate the Rx signal as well. In general, the three time domain currents $i(t), \mathrm{d} i / \mathrm{d} t$, and $\int \mathrm{d} t i(t)$ might be present, which complicates considerably the design of the optimum Rx. Note that even though it is possible in principle to design a circuit that can extract $i(t)$ from the total Rx signal (19), to do so one must effectively solve a differential equation at the receiver. The main difficulty in this case is that the coefficients of the three terms $i(t), \mathrm{d} i / \mathrm{d} t$, and $\int \mathrm{d} t i(t)$ depend on the position of the $\mathrm{Rx}$.

The common practice in wireless communications research has been to design the digital receiver based on far-field scenarios since this provides the most general case and can be adapted later to NFC links [50]. If this strategy is adopted, terms containing $i(t)$ and $\int \mathrm{d} t i(t)$, which originate from the impinging near-field components, are bound to produce observable distortion in the Rx signal. Distortion, a deterministic effect, is another factor besides noise that degrades the performance of digital communication links and contributes toward the increase of the probability of error in the digital link [51]. Note that in recent $4 \mathrm{G}$ and $5 \mathrm{G}$ systems, the wide deployment of massive MIMO [52,53] technologies and increasingly denser networks have made the importance of near-field contributions more significant than before [54]. In Sec. 3, we will remove the distortion caused by the NF current term (21) using NSAs by finding a specialized current pulse to use in for digital data transmission.

Putting aside for a while the relative contribution of each of the three waveforms $i(t), \mathrm{d} i / \mathrm{d} t$, and $\int \mathrm{d} t i(t)$, we note further from (19), (20), (21) that each such time signal is filtered through the receiving antenna impulse response $F\left(\mathbf{r}_{p}, t\right)$. Such filtration will produce a distortion since it contributes to the broadening of the Rx signal pulse [55], [56], [57]. More specifically, the generic form of the Rx signal

${ }^{\dagger}$ But this current is evaluated at the retarded time $t_{r}$. 
$i_{\text {rx }}(t)$, which is defined by

$$
i_{\mathrm{rx}}(t):=\hat{\alpha} \cdot \mathbf{J}\left(\mathbf{r}_{p}, t\right),
$$

turns out to be given by a general expression of the form

$$
i_{\mathrm{rx}}(t)=\underbrace{\frac{a_{1}}{r} F(t) * \frac{\mathrm{d} i\left(t_{r}\right)}{\mathrm{d} t}}_{\text {Far-field signal }}+\underbrace{\frac{a_{2}}{r^{2}} F(t) * i\left(t_{r}\right)+\frac{a_{3}}{r^{3}} F(t) * \int \mathrm{d} t_{r} i\left(t_{r}\right)}_{\text {Near-field induced distortion }}+\underbrace{n(t)}_{\text {Noise }},
$$

where for simplicity the small antenna impulse response $F\left(\mathbf{r}_{p} ; t\right)$ was written as $F(t)$, i.e., without $\mathbf{r}_{p}$ provided the location of the Rx port is clear from the context. All remaining factors in (19), (20), (21) were collected into the coefficients $a_{1}, a_{2}, a_{3}$ given by

$$
a_{1}:=\frac{Z_{0} l}{4 \pi c} \hat{\alpha} \cdot(\hat{r} \times \hat{r} \times \hat{l}), \quad a_{2}:=\frac{Z_{0} l}{4 \pi c}\{[\hat{r} \times \hat{r} \times \hat{l}+2(\hat{l} \cdot \hat{r}) \hat{r}] \cdot \hat{\alpha}\}, \quad a_{3}:=c a_{2} .
$$

A wide-sense stationary zero-mean thermal noise process $n(t)$ was also inserted into (24) in order to account for random fluctuations in the environment and the receiver noise [50,51].

Next, in order to remove the effect of the antenna filtration, an equalizer is introduced. Formally, the equalizer is an LTI filter that has a response function inverse to that of the antenna $F(t)[51]$. Let the impulse response of the equalizer be $F_{\text {eq }}(t)$. We then require that

$$
F(t) * F_{\text {eq }}(t)=\delta(t),
$$

where $\delta(t)$ is the Dirac delta function. The design of an equalizer circuit by means of the ideal condition (26) is not always practically feasible, and for various reasons. First, if we examine the frequency-domain version of (26), the following must be satisfied

$$
F_{\text {eq }}(\omega)=\mathcal{F}^{-1}\left\{\frac{1}{F(\omega)}\right\}
$$

where $\mathcal{F}^{-1}$ is the inverse of the Fourier transform operator $\mathcal{F}$, while $F(\omega)$ and $F_{\text {eq }}(\omega)$ are the Fourier transforms of $F(t)$ and $F_{\text {eq }}(t)$, respectively. It can be inferred from (27) that when the forward gain $F(\omega)$ is very small, the inverse filter gain may become very large, leading to instability. Other considerations include the possibility that by striving to satisfy (26), the inverse filter may become noncausal. For these reasons, in practice digital equalizers like Zero-Forcing (ZF) and MMSE equalizers are often used to remove the channel effect $[51,52]$. This, however, is most often applied to the distortion caused by multipath channels [50,53]. In our basic NFC system, multipath fading is ignored for simplicity. Instead, distortion is here caused mainly by the electromagnetic response of the antenna device itself, as captured by the impulse response $F(t)$. Recently, the idea of using digital equalization techniques like OFDM to solve this problem were proposed and demonstrated that the digital transmission data rate supported by a SISO line-of-sight deploying half-wavelength dipole antennas could be increased by up to three times the data rate supported by the matching bandwidth [55], [57], [58]. For our purposes in this paper the exact details of the equalizer design are irrelevant to the main subject (and can always be dealt with in a more specialized papers elsewhere), so we assume that an ideal equalizer has been constructed and applied to the Rx signal $i_{\mathrm{rx}}(t)(24)$, leading to the equalized signal

$$
i_{\mathrm{eq}}(t)=\underbrace{\frac{a_{1}}{r} \frac{\mathrm{d} i\left(t_{r}\right)}{\mathrm{d} t}}_{\text {Far-field signal }}+\underbrace{\frac{a_{2}}{r^{2}} i\left(t_{r}\right)+\frac{a_{3}}{r^{3}} \int \mathrm{d} t_{r} i\left(t_{r}\right)+F_{\mathrm{eq}}(t) * n(t)}_{\text {Distortion (near-field contribution) }+ \text { thermal noise }},
$$

where both filtered noise and the NF terms are collected together. Finally, by passing the equalized signal through an integrator, the following signal is produced

$$
i_{\text {eq }}(t)=\underbrace{\frac{a_{1}}{r} i\left(t_{r}\right)}_{\text {Desired signal }}+\underbrace{\frac{a_{2}}{r^{2}} \int \mathrm{d} t_{r} i\left(t_{r}\right)+\frac{a_{3}}{r^{3}} \int \mathrm{d} t_{r}\left[\int \mathrm{d} t_{r} i\left(t_{r}\right)\right]+\int \mathrm{d} t_{r} F_{\text {eq }}(t) * n(t)}_{\text {Undesired signal: Distortion (NF contribution) }+ \text { thermal noise }} .
$$


In other words and to express the same result using standard digital communications literature, the total current sampled by the receiver is given by

$$
i_{\mathrm{eq}}(t)=x(t)+d(t)+w(t)
$$

where

$$
x(t):=\frac{a_{1}}{r} i\left(t_{r}\right), d(t):=\frac{a_{2}}{r^{2}} \int \mathrm{d} t_{r} i\left(t_{r}\right)+\frac{a_{3}}{r^{3}} \int \mathrm{d} t_{r}\left[\int \mathrm{d} t_{r} i\left(t_{r}\right)\right], w(t):=\int \mathrm{d} t_{r} F_{\text {eq }}(t) * n(t),
$$

are the desired signals, the NF-induced distortion signal, and filtered noise signals, respectively. Because we work with physical spacetime fields only in this paper (causal and finite-energy signals), the traditional signal energy

$$
\mathcal{E}_{x}:=\int_{-\infty}^{\infty} \mathrm{d} t x(t)^{2}
$$

can be used instead of power to estimate the "size" of a given deterministic waveform. Since the distortion signal $d(t)$ is also deterministic, its energy can very well be estimated by the identical formula

$$
\mathcal{E}_{d}:=\int_{-\infty}^{\infty} \mathrm{d} t d(t)^{2}
$$

On the other hand, the noise "size" can be estimated by

$$
\mathcal{E}_{w}:=\mathbb{E}\left\{w(t)^{2}\right\},
$$

where $\mathbb{E}$ is the expected value operator [51]. Based on these definitions, the Signal-Noise-Ratio (SNR) can be computed via

$$
\mathrm{SNR}:=\frac{\mathcal{E}_{x}}{\mathcal{E}_{n}},
$$

while the Signal-to-Noise-and-Interference (SNIR) is found by means of

$$
\operatorname{SNIR}:=\frac{\mathcal{E}_{x}}{\mathcal{E}_{d}+\mathcal{E}_{w}} .
$$

Here, by 'interference' we refer to the distortion signal $d(t)$ that interferes with the far-field (desired) signal $x(t)$. It is plain now that due to the presence of NF components in NFC systems and even modern day massive MIMO environments, the energy $\mathcal{E}_{d}$ may become significant compared with $\mathcal{E}_{w}$, leading to

$$
\mathrm{SNIR} \leq \mathrm{SNR} .
$$

In far-field communications or when NF effects are negligible, we have SNIR $\approx \mathrm{SNR}$.

\section{ENGINEERING THE NEAR-FIELD STRUCTURE USING SPECIALIZED TIME-DOMAIN EXCITATION}

Our goal now is to show that NSAs provide a new engineering dimension not present in traditional sinusoidal antennas, a novel potential opened up by the ability to fully control the structure of the radiated near fields through careful manipulations of the time-waveform functions applied at the antenna input terminals. Our starting point will be the observation arrived at in Sec. 2 where the NF signal $d(t)$ appearing in the total Rx signal (30) presents the main source of possible distortion in NFC links. By inspecting the mathematical expression of $d(t)$ given in (31), we notice that the functional time variations of the excitaion current $i(t)$ can make $d(t)$ vanish. That is, we set

$$
d(t)=\frac{a_{2}}{r^{2}} \int \mathrm{d} t_{r} i\left(t_{r}\right)+\frac{a_{3}}{r^{3}} \int \mathrm{d} t_{r}\left[\int \mathrm{d} t_{r} i\left(t_{r}\right)\right]=0 .
$$


Differentiating twice, we arrive at the following first-order differential equation

$$
\frac{a_{2}}{r^{2}} \frac{\mathrm{d} i\left(t_{r}\right)}{\mathrm{d} t_{r}}+\frac{a_{3}}{r^{3}} i\left(t_{r}\right)=0
$$

which can be rewritten in the standard form

$$
\frac{\mathrm{d} i(t)}{\mathrm{d} t}+\frac{a_{3}}{a_{2} r} i(t)=0
$$

The relation (40) is the main differential equation that must be satisfied by the current exciting the small NSA if the near-field signal $d(t)$ is to vanish. It is clearly dependent on the distance from NSA $r$ at which the near field is required to nullify. Since we are intending to analyze a NFC system where the $\mathrm{Rx}$ antenna is set at a fixed distance from the $\mathrm{Tx}$, we may take $r$ appearing in (40) as constant, denoted by $r_{c}$ in what follows.

The solution of (40) can be readily found and is simply the exponential function

$$
i(t)=i_{0} e^{-t / \tau},
$$

where $i_{0}$ and $\tau$ are real constants. The value of $i_{0}$ is determined by the NSA source while the time constant $\tau$ is fixed to

$$
\tau:=\frac{-a_{2} r_{s}}{a_{3}},
$$

which is a function of the Rx distance $r_{c}$. From (25), we may determine

$$
\tau=\frac{r_{s}}{c} .
$$

The relation (43) is interesting and suggests that a very special current can produce zero near-field at the sphere $r=r_{s}$, i.e., that case when the NSA is excited by an exponentially decaying signal with the form (41). It is easy to see that no sinusoidal-like excitation satisfies the differential equation above and hence the cancellation of the NF at the sphere $r=r_{s}$ is a unique feature enjoyed only by NSAs.

Let us explore some consequences of exciting the NSA with a current source having the form given in (41). It is clear that by the construction (38), the total radiated field (9) when evaluated at $\mathbf{r}=\mathbf{r}_{s}$ will yield

$$
\mathbf{E}\left(\mathbf{r}_{s}, t\right)=\frac{Z_{0} l}{4 \pi c}\left\{\frac{1}{r_{s}} \frac{\mathrm{d} i\left(t_{r}\right)}{\mathrm{d} t} \hat{r} \times \hat{r} \times \hat{l}\right\}
$$

which is composed of far field only. By inserting the special current (41) into (44), the following expression is obtained

$$
\mathbf{E}\left(\mathbf{r}_{s}, t\right)=\frac{-Z_{0} l}{4 \pi r_{s}^{2}}(\hat{r} \times \hat{r} \times \hat{l}) e^{-t / \tau}
$$

In other words, even deep into the near-field zone, an NSA excited by a decaying current pulse with time constant equal to the time needed to reach the sphere $r=r_{s}$ in the NF zone will produce zero near field on that sphere. Note that this remarkable result is valid only at the sphere $r=r_{s}$; in fact, immediately before and after this sphere, a near-field component will quickly build up.

Consider next the magnetic field with its general expression given in (10). At the sphere $r=r_{s}$, the total magnetic field is

$$
\mathbf{H}\left(\mathbf{r}_{s}, t\right)=\frac{l}{4 \pi c}\left(\frac{1}{r_{s}} \frac{d i\left(t_{r}\right)}{d t}+\frac{c}{r_{s}^{2}} i\left(t_{r}\right)\right) \hat{l} \times \hat{r} .
$$

If we substitute (41) into the last relation, it turns out that the magnetic field is identically zero, i.e., we have

$$
\mathbf{H}\left(\mathbf{r}_{s}, t\right)=0 .
$$

In other words, while only the near-field component of $\mathbf{E}\left(\mathbf{r}_{s}, t\right)$ is zero on the sphere $r=r_{s}$, it turns out that in that special case the total (i.e., NF + FF components) magnetic field vanishes on the same 
sphere. One immediate corollary to (47) is that the instantaneous Poynting vector $\mathbf{S}(\mathbf{r}, t)$ is also zero on the sphere $r=r_{s}$, that is,

$$
\mathbf{S}\left(\mathbf{r}_{s}, t\right):=\mathbf{E}\left(\mathbf{r}_{s}, t\right) \times \mathbf{H}\left(\mathbf{r}_{s}, t\right)=0 .
$$

Again we note that this remarkable zeroing out of the Poynting flow occurs only at a single sphere. Just before and after $r=r_{s}$, the Poynting flow is nonzero (although it changes sign when crossing the critical sphere). This zeroing out of Poynting flow appears to have been first observed by Schantz in $[1,59,60]$, though from a different scope and perspective of the present work.

It is important to note that the Rx antenna can still capture EM energy even if the Poynting flow is critically zero at the reception sphere $r=r_{s}$. The reason is that the induced current on the Rx antenna depends on the strength of the dynamic electric field (the magnetic field does not interact with PEC objects.) It can be shown using the exact ACGF formula (11) that the power delivered to the load in the Rx system is proportional to the correlation function of the incident electric field, not the Poynting vector [61]. This is also clear from the physics of PEC antennas in the receive mode since currents are introduced by direct force exerted by the electric field on the conduction band electrons via Lorentz force law (the Poynting vector does not appear in this law) [33].

\section{GENERAL REMARKS ON REALIZATION AND APPLICATIONS OF NONSINUSOIDAL ANTENNAS}

Mainstream antennas tend to rely on excitation methods where a transmission line is used to convey the generator signal to the antenna [36]. However, NSAs can operate by directly connecting the generator to the antenna terminals if special circuits (driver circuitry with active switches) are carefully designed to orchestrate the energy exchange cycles taking place between the antenna and the generator (driver) system. Not much research has been conducted on the experimental realization of nonsinusoidal antenna since frequency-domain devices now dominate the literature. However, there are a few exceptions (to the best of our knowledge.) Various circuit designs for the excitation of NSAs where given in [62-66]. More recently, it was shown numerically and experimentally that the design of highly specialized time-domain excitation circuits in ESAs can lead to antennas radiating data rates well above what is expected from the narrow-band frequency-domain matching bandwidth of resonant antennas like ESAs [67], [68], [69], [70]. This appears to be essentially the insight or goal that motivated Harmuth and his collaborators originally to take up the subject in the 1970s and 1980s since they were primarily interested in applications to digital communication, radar, and sensor systems. That is, the more recent research in addition to the ideas presented in this paper strongly suggest that the subject of nonsinusoidal antennas is interesting and worth investigating.

Based on the overall theory presented in this paper, we propose building complete near-field digital communication systems using NSAs as transmitters and receivers. In Fig. 1, we provide an overall sketch of the architecture. As mentioned above, the NSA system consists of jointly designed driver circuit and the radiator unit. The goal is to produce the specialized pulse current $i(t)$ that will be able to attain zero near-field at the distance $R=c \tau$. In practice, the hardware design aspects (which are not covered in this paper) requires synchronising the energy exchange cycles between the radiator and the driver as per some of the published implementation literature cited above. From the fundamental electromagnetic and communication viewpoint (the main subject of this paper), we have stressed the importance of placing the small receive prove in such a position such that the total near field is zero, leading to considerable simplification in the equalizer design in the receiver side since distortion caused by near-field components will not be present and traditional, off-the-shelf equalizers based on only farfield propagation assumption can still be used. The NFC system will send a series of basic pulses $i(t)$, i.e., the transmitted signal $i_{\mathrm{tx}}(t)$ is given by

$$
i_{\mathrm{tx}}(t)=\sum_{n} i(t-n T),
$$

where $T=1 / \mathrm{R}$, where $\mathrm{R}$ is the data rate in symbol per second. In other words, each digital symbol will be encoded by a specialized decaying pulse of idealized form (50) or the practical approximation (52) but assuming $T>\tau$. Intersymbol interference (ISI), which is bound to happen in distorting links, 
will be minimized when the Rx NSA is placed at the exact zero-NF sphere $r=r_{s}$, leading to superior performance. The detailed analysis of such systems with efficient modulation schemes such as QAMOFDM $[51,53]$ requires estimating the spectral efficiency and is beyond the scope of this paper but will be addressed in the future.

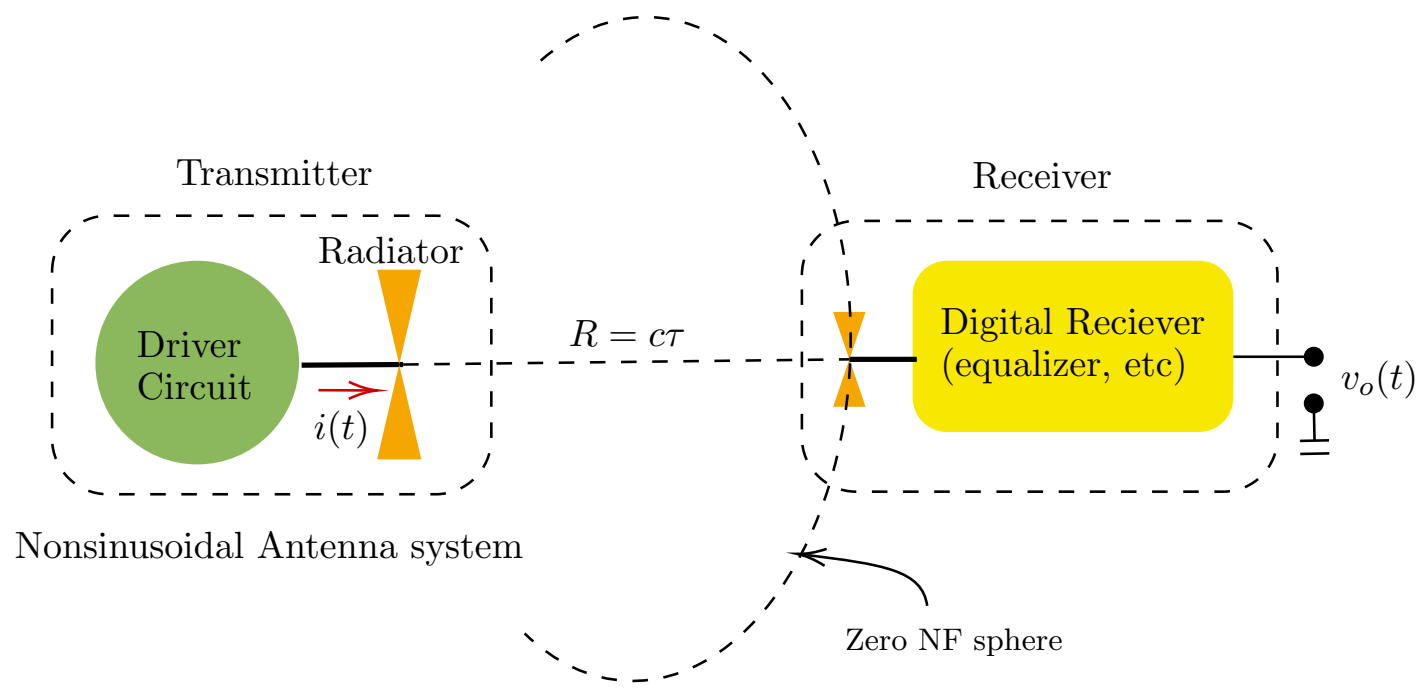

Figure 1: A general near-field communication system utilizing a nonsinusoidal antenna (NSA) transmitter. The NSA itself is composed of a driver circuit and a radiator. In contrast to mainstream sinusoidal antennas, the driver circuit, which produces the specialized nonsinusoidal signal $i(t)$, must be designed jointly with the radiator itself. The Receiver is composed of a receive probe connected to digital reeciever that includes an equalizer and other signal processing blocks.

The decaying current excitation in (41), however, is not fully physically realizable because of the need to consider what happens before switching on the current source. Since the antenna must be charged to the level where the current will start at the maximum value $i_{0}$ at $t=0$, one must explicitly include in the analysis radiation caused by the rising edge of the excitation current $i(t), t<0$. Since this part of the rising current does not satisfy the main differential equation (40), residual near-field radiation is expected to be present at the NFC sphere $r=r_{s}$. However, once the current begins to decay according to (41), the EM field settles back to the predictions made in (44) and (47). For example, let us assume that the effective excitation is

$$
i(t)=\left\{\begin{array}{l}
i_{0} e^{-t / \tau}, \quad t \geq 0 \\
0, \quad \text { otherwise. }
\end{array}\right.
$$

In this case, after inserting such current into the radiation formulas (9) and (10), a Dirac delta component will be generated, producing very high values near the considerably lower levels of the radiation produced by the falling edge of the excitation pulse. To avoid this problem, we can introduce especially engineered pulses with tailored rising edges designed to minimize specific effects (This method is commonly used in UWB antenna engineering [1].) As a very basic and simple choice, consider the case when we derive the NSA such that the charge follows the form

$$
q(t)=\frac{1}{\exp (t / \tau)+\exp (-t / \sigma)},
$$

where it is assumed that $\sigma \ll \tau$. For $t>0$, the decaying exponential $\exp (-t / \sigma)$ can be neglected in comparison to the growing $\exp (t / \tau)$, so the denominator is essentially dominated by the latter, making (51) approximately equal to $\exp (-t / \tau)$. On the other hand, for $t<0$, the condition $\sigma \ll \tau$ and the fact that the role of growing and decaying exponentials are reserved makes the increasing function $\exp (-t / \sigma)$ dominant in the denominator, creating then a rising edge since $q(t)$ becomes approximately equal to 


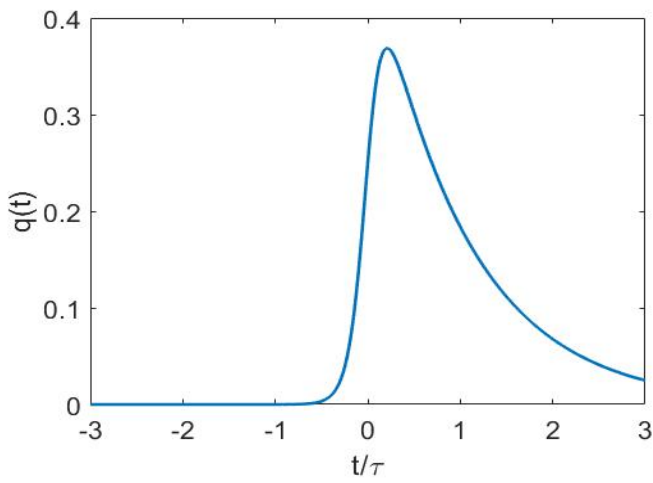

(a) Charge pulse

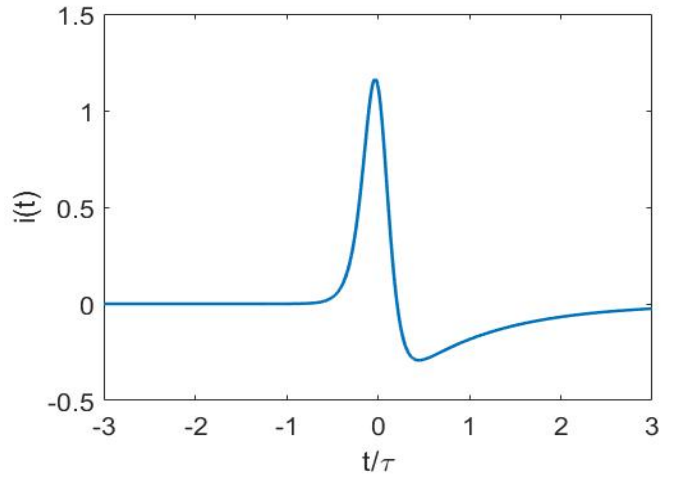

(b) Current pulse

Figure 2: The charge and current pulses based on the approximating finite-rise time source functions (51) and (52).

$\exp (t / \sigma)$. Next, the corresponding current is computed as

$$
i(t)=\frac{\mathrm{d} q(t)}{\mathrm{d} t}=\frac{-(1 / \tau) \exp (t / \tau)+(1 / \sigma) \exp (-t / \sigma)}{[\exp (t / \tau)+\exp (-t / \sigma)]^{2}} .
$$

Without the need for any new analysis, we note that since $q(t)$ as given by (51) behaves like a decaying exponential for $t>0$ and a growing exponential when $t<0$, then so will be its derivative, with the only (unimportant) difference being a sign change suffered by the amplitudes of the respective exponentials. Finally, since both (51) and (52) are smooth (infinitely differentiable), we conclude that the current excitation (52) provides a practical smooth driving source with good approximation of the fundamental solution (decaying current) obtained in (41), e.g., see Figs. 2(a) and 2(b).

Unfortunately, if the modified expressions (51) and (52) are used instead of the exact solution (50), no entire-time exact cancellation of the near field can be attained at a fixed radius. Instead, a new location for zero near field can be found but it works only for fixed time instants. In fact, it is easy to see that the receiver in this case must be located at a time-varying position given by the distance $r_{s}^{\prime}$, where

$$
r_{s}^{\prime}=\tau_{m} c, \quad \tau_{m}=\frac{\exp (t / \tau)+\exp (-t / \sigma)}{(1 / \tau) \exp (t / \tau)+(1 / \sigma) \exp (-t / \sigma)}
$$

which can be readily verified by noting that (53) forces the near-field radiation $\mathbf{E}_{\mathrm{nf}}(\mathbf{r}, t)$ to be zero, where the following expression (which can be obtained from (9))

$$
\mathbf{E}_{\mathrm{nf}}(\mathbf{r}, t)=\frac{Z_{0} l}{4 \pi c}\left(\frac{c}{r^{2}} i\left(t_{r}\right)+\frac{c^{2}}{r^{3}} q(t)\right)[\hat{r} \times \hat{r} \times \hat{l}+2(\hat{l} \cdot \hat{r}) \hat{r}]
$$

is used to prove this theorem. The near-field pulse at the critical distance is shown in Fig. 3(a) where the distance $r$ in (54) is given by the exact expression (43) instead of the modified distance (53). It is clear that the near field fails to fully cancel at all time instants as predicted above. In Fig. 3(b) we show the time variations of the distance between the Tx and Rx in the proposed NF communication system. As we can see, ideally one must change the location of the receiver with time to ensure the near field is always zero, i.e., for all time instants. On the other hand, one may fix the Rx probe but in this case cancellation of the near field is ensured at only one time instant. This is not very bad given that in most modern digital communications only the sample of the received signal at a specific switching time is taken by the digital receiver. Therefore, it appears that a joint planning of the digital receiver and the available electromagnetic parameters like position and timing of the receiving end is called for. Consequently, optimization is needed when working with practical systems. In our case, the values of $\tau$ and $\sigma$ must be chosen very carefully, depending on where and how the receive antenna will be installed. 


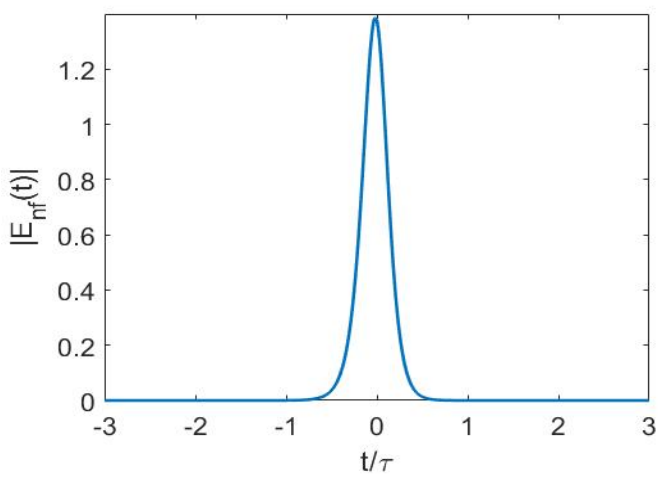

(a) Near-field pulse at $r=r_{s}$ (normalized to $Z_{0} l /(4 \pi c)$.)

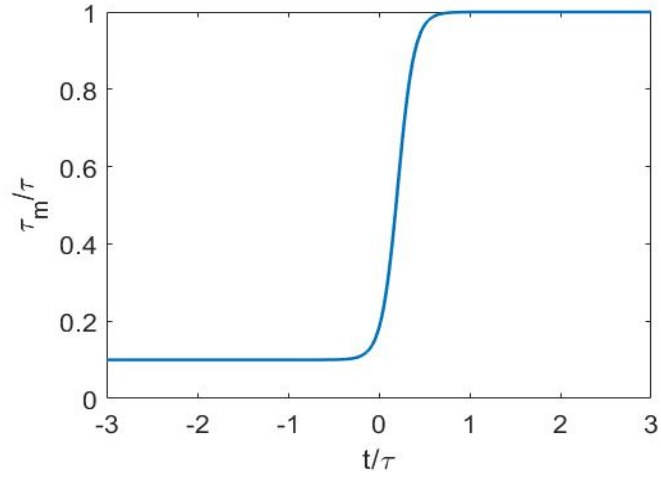

(b) Modified receiver time distance $\tau_{m}$.

Figure 3: Analysis of the impact of using non-exact pulse excitation in the NF communication link. (a) The broadside $(\hat{l} \cdot \hat{r}=0)$ near field amplitude computed using the practical pulse excitation in Figs. 2 (a) and 2(b) is computed by means of the exact field expression (54) with the ideal expression $r_{s}=c \tau$ inserted for the position $r$. Here, the amplitude is normalized with respect to the peak value $Z_{0} l /(4 \pi c)$. (b) The corrected time distance between the Tx and $\mathrm{Rx}$ in order to cancel the near field. If the $\mathrm{Rx}$ is placed at a sphere with time-varying radius given by $r_{s}^{\prime}=c \tau_{m}$, the near field is always zero.

Such optimization requires careful and intensive numerical approach that is outside the scope of the present paper, which is mainly concerned with the fundamental ideas and their proof of concept.

Another promising application of small NSAs is in the field of nanoscale optical communications. Since our engineered NSA excited by a decaying pulse can communicate well at short distances, they can be deployed in situations requiring very short-range transfer of information or power. Nanoscale environments tend to have extremely lossy and hazardous surroundings, forcing many attempts to send signals using light effectively short ranged, not to mention the weakness of the transmitted signal due to the smallness of the scattering cross section of nanoscale radiating systems $[38,71,72]$. However, the realization of the specialized decaying pulse as a possible information carrier is quite feasible in the nanoscale since quantum emitters, operating through the phenomenon of spontaneous emission of photons, can be modeled very well by decaying pulses of the form derived above. However, controlling when the decay occurs and the time constant $\tau$ remains challenging though considerable progress has been made in recent years [38].

Finally, we add that the theory presented in this paper can be expanded to include larger antennas by aggregating the effects of individual infinitesimal dipoles using the Infinitesimal Dipole Model (IDM) technique, which is an electromagnetic machine learning capable of learning from NF or FF data the best small dipole model capable of representing radiation everywhere in the exterior domain [73-81]. The details of this expansion will be the subject of future work.

\section{CONCLUSION}

A general theoretical framework for a class of radiators called nonsinusoidal antennas was developed with emphasis on the special case when the antenna can be considered small. It was found that an ordinary differential equation can be derived which when solved yields the exact form of a timedependent but inherently nonsinusoidal excitation current such that the radiated near-field is exactly zero on a particular sphere near the antenna. In this way, NF communication systems can be designed and build with no NF-induced distortion effect. The concept has been developed carefully mainly at the conceptual and theoretical level, with the various stages in the digital receiver design developed using exact Green's function technique. Some issues pertinent to future realization and application of the proposed system where also briefly discussed. It is the hope of the author that this paper will bring to the attention of the antenna community the enormous potentials available to physicists and engineers in the time domain. Time-domain or nonsinusoidal antennas present a large field of research that has 
not not be well explored so far but is expected to gain more importance as we move into the future with applications like $6 \mathrm{G}$ and nanoscale communication and information processing.

\section{REFERENCES}

1. H. Schantz, The art and science of ultrawideband antennas. Boston: Artech House, 2015.

2. H. Harmuth and M. Hussain, Propagation of electromagnetic signals. Singapore River Edge, N.J: World Scientific, 1994.

3. Jun Zhan and Qi-Ling Qin, "Analytic solution of traveling-wave antennas excited by nonsinusoidal currents," IEEE Transactions on Electromagnetic Compatibility, vol. 31, no. 3, pp. 328-330, Aug 1989.

4. H. F. Harmuth, "Radar equation for nonsinusoidal waves," IEEE Transactions on Electromagnetic Compatibility, vol. 31, no. 2, pp. 138-147, May 1989.

5. H. F. Harmuth and S. Ding-Rong, "Antennas for nonsinusoidal waves. I. radiators," IEEE Transactions on Electromagnetic Compatibility, vol. EMC-25, no. 1, pp. 13-24, Feb 1983.

6. — , "Antennas for nonsinusoidal waves: II-sensors," IEEE Transactions on Electromagnetic Compatibility, vol. EMC-25, no. 2, pp. 107-115, May 1983.

7. H. Harmuth, Antennas and waveguides for nonsinusoidal waves. Orlando: Academic Press, 1984.

8. H. F. Harmuth, "Radiation of nonsinusoidal waves by a large-current radiator," IEEE Transactions on Electromagnetic Compatibility, vol. EMC-27, no. 2, pp. 77-87, May 1985.

9. H. F. Harmuth, N. J. Mohamed, and R. N. Boules, "Power flowing through the surface of a largecurrent radiator," IEEE Transactions on Electromagnetic Compatibility, vol. 28, no. 3, pp. 131-141, Aug 1986.

10. H. F. Harmuth and N. J. Mohamed, "Large-current radiators," IEE Proceedings H - Microwaves, Antennas and Propagation, vol. 139, no. 4, pp. 358-362, Aug 1992.

11. H. F. Harmuth, "Correction of maxwell's equations for signals I," IEEE Transactions on Electromagnetic Compatibility, vol. 28, no. 4, pp. 250-258, Nov 1986.

12. — - "Correction of maxwell's equations for signals II," IEEE Transactions on Electromagnetic Compatibility, vol. 28, no. 4, pp. 259-266, Nov 1986.

13. A. Lakhtakia, "Nonuniqueness of the inverse laplace transform, and the debate on Harmuth's technique," IEEE Transactions on Electromagnetic Compatibility, vol. 36, no. 3, pp. 256-258, Aug 1994.

14. H. F. Harmuth, "Propagation velocity of electromagnetic signals," IEEE Transactions on Electromagnetic Compatibility, vol. 28, no. 4, pp. 267-272, Nov 1986.

15. H. Harmuth, Propagation of nonsinusoidal electromagnetic waves. Orlando: Academic Press, 1986.

16. W. Geyi, Foundations of Applied Electrodynamics. Chichester, West Sussex Hoboken, N.J: Wiley, 2010.

17. S. Mikki, D. Sarkar, and Y. Antar, "Beyond antenna Q: On reactive energy and the need for a spatio-temporal dynamical paradigm," in 2019 13th European Conference on Antennas and Propagation (EuCAP), March 2019, pp. 1-5.

18. D. Sarkar, S. Mikki, A. Alzahed, K. V. Srivastava, and Y. Antar, "New considerations on electromagnetic energy in antenna near-field by time-domain approach," in 2017 IEEE Applied Electromagnetics Conference (AEMC), Dec 2017, pp. 1-2.

19. D. Sarkar, S. Mikki, K. V. Srivastava, and Y. Antar, "Dynamics of antenna reactive energy using time-domain IDM method," IEEE Transactions on Antennas and Propagation, vol. 67, no. 2, pp. 1084-1093, Feb 2019.

20. A. Alzahed, S. Mikki, and Y. Antar, "Stored energy in general antenna system: A new approach," in 2016 10th European Conference on Antennas and Propagation (EuCAP), April 2016, pp. 1-5.

21. T. Hansen, Plane-Wave Theory of Time-Domain Fields: Near-Field Scanning applications. New York: IEEE Press, 1999. 
22. S. Mikki and Y. Antar, "A theory of antenna electromagnetic near field-part I," IEEE Transactions on Antennas and Propagation, vol. 59, no. 12, pp. 4691-4705, December 2011.

23. S. M. Mikki and Y. M. M. Antar, "A theory of antenna electromagnetic near field - part II," IEEE Transactions on Antennas and Propagation, vol. 59, no. 12, pp. 4706-4724, Dec 2011.

24. S. Mikki, D. Sarkar, and Y. Antar, "Near-field cross-correlation analysis for MIMO wireless communications," IEEE Antennas and Wireless Propagation Letters, vol. 18, no. 7, pp. 1357-1361, July 2019.

25. M. A. Nikravan, D. Kwon, H. G. Schantz, and A. H. Unden, "Near-field MIMO communication utilizing both electric and magnetic field components," in 2014 IEEE Antennas and Propagation Society International Symposium (APSURSI), July 2014, pp. 474-475.

26. S. Phang, M. T. Ivrlac, G. Gradoni, S. C. Creagh, G. Tanner, and J. A. Nossek, "Near-field MIMO communication links," IEEE Transactions on Circuits and Systems I: Regular Papers, vol. 65, no. 9, pp. 3027-3036, 2019.

27. Y. S. Chen, S. Y. Chen, and H. J. Li, "Analysis of Antenna Coupling in Near-Field Communication Systems," IEEE Transactions on Antennas and Propagation, vol. 58, no. 10, pp. 3327-3335, 2010.

28. Y. Tak and S. Nam, "Mode-based computation method of channel characteristics for a near-field MIMO," IEEE Antennas and Wireless Propagation Letters, vol. 10, pp. 1170-1173, 2011.

29. H.-J. Kim, J. Park, K.-S. Oh, J. P. Choi, J. E. Jang, and J.-W. Choi, "Near-field magnetic induction MIMO communication using heterogeneous multipole loop antenna array for higher data rate transmission," IEEE Transactions on Antennas and Propagation, vol. 64, no. 5, pp. 1952-1962, 2016.

30. S. M. Mikki and Y. M. M. Antar, "A new technique for the analysis of energy coupling and exchange in general antenna systems," IEEE Transactions on Antennas and Propagation, vol. 63, no. 12, pp. 5536-5547, Dec 2015.

31. S. Clauzier, S. Mikki, and Y. Antar, "Design of near-field synthesis arrays through global optimization," IEEE Transactions on Antennas and Propagation, vol. 63, no. 1, pp. 151-165, Jan 2015.

32. S. Mikki, "The antenna spacetime system theory of wireless communications," Proceedings of the Royal Society A: Mathematical, Physical and Engineering Sciences, April 2019.

33. J. Schwinger et al., Classical electrodynamics. Reading, Mass: Perseus Books, 1998.

34. A. Zangwill, Modern electrodynamics. Cambridge: Cambridge University Press, 2013.

35. A. Garg, Classical electromagnetism in a nutshell. Princeton N.J: Princeton University Press, 2012.

36. C. A. Balanis, Antenna Theory: Analysis and Design, 4th ed. Inter-science: Wiley, 2015.

37. O. Keller, Quantum Theory of Near-Field Electrodynamics. Berlin New York: Springer, 2011.

38. L. Novotny, Principles of Nano-Optics. Cambridge: Cambridge University Press, 2012.

39. S. Mikki and Y. M. Antar, "Analysis of electromagnetic interactions in antenna arrays using the antenna current Green's function method," in Proceedings of IEEE APS-URSI International Symposium, 2011, pp. 3-8.

40. S. Mikki and Y. Antar, "On the Fundamental Relationship Between the Transmitting and Receiving Modes of General Antenna Systems: A New Approach," IEEE Antennas and Wireless Propagation Letters, vol. 11, pp. 232-235, 2012.

41. S. Mikki and Y. Antar, "The antenna current Green's function as an alternative method to conventional full-wave analysis solvers: An outline," in 2015 IEEE MTT-S International Conference on Numerical Electromagnetic and Multiphysics Modeling and Optimization (NEMO), Aug 2015, pp. 1-3.

42. S. Mikki and Y. Antar, "The antenna current Green's function formalism-Part I," IEEE Trans. Antennas Propagat, vol. 9, pp. 4493-4504, September 2013.

43. — - "The antenna current Green's function formalism-Part II," IEEE Trans. Antennas Propagat, vol. 9, pp. 4505-4519, September 2013.

44. —_ "Physical And Computational Aspects Of Antenna Near Fields: The Scalar Theory," Progress 
In Electromagnetics Research B, vol. 63, p. 6778, 2015.

45. - "Analysis of generic near-field interactions using the antenna current Green's function," Progress of Electromagnetic Research C (PIER C), vol. 59, pp. 1-9, 2015.

46. — New Foundations for Applied Electromagnetics: The Spatial Structure of Fields. London: Artech House, 2016.

47. — - "Fundamental Research Directives in Applied Electromagnetics," in 2011 28th National Radio Science Conference (NRSC), April 2011, pp. 1-9.

48. S. Mikki and Y. Antar, "Unifying electromagnetic and communication theories: A proposal for a new research program," in 2016 URSI International Symposium on Electromagnetic Theory (EMTS), Aug 2016, pp. 435-438.

49. I. M. Gelfand and G. E. Shilov, Generalized functions (volume I). Providence, Rhode Island: American Mathematical Society AMS Chelsea Publishing (re-print edition), 2016.

50. T. S. Rappaport, R. H. Jr, R. C. Daniels, and J. N. Murdock, Millimeter Wave Wireless Communications. Upper Saddle River, N.J: Prentice Hall, 2014.

51. B. P. Lathi and Z. Ding, Modern digital and analog communication systems. New York: Oxford University Press, 2019.

52. J. Hampton, Introduction to MIMO communications. Cambridge: Cambridge University Press, 2013.

53. R. Heath, Foundations of MIMO communication. Cambridge, United Kingdom New York, NY, USA: Cambridge University Press, 2019.

54. Y. Yang, J. Xu, G. Shi, and C.-X. Wang, 5G Wireless Systems: Simulation and Evaluation Techniques. Springer, 2017.

55. S. Mikki, A. Hanoon, J. Aulin, and Y. Antar, "The time-dependent ACGF with applications to M-ary digital communication systems," in The 11th European Conference on Antennas and Propagation (EuCap 2017), 2017, pp. 19-24.

56. S. Mikki, A. Hanoon, J. Persano, A. Alzahed, Y. Antar, and J. Aulin, "Theory of electromagnetic intelligent agents with applications to MIMO and DoA systems," in 2017 IEEE International Symposium on Antennas and Propagation USNC/URSI National Radio Science Meeting, July 2017, pp. $525-526$.

57. A. Hanoon and S. Mikki, "Bandwidth-enhancement of digital communication systems employing narrowband antennas: A novel electromagnetic OFDM approach," in 2017 IEEE International Symposium on Antennas and Propagation USNC/URSI National Radio Science Meeting, July 2017, pp. 527-528.

58. A. Hanoon, Electromagnetic OFDM for Wireless Digital Communication System. University of New Haven: MS Thesis, 2017.

59. H. G. Schantz, "The flow of electromagnetic energy in the decay of an electric dipole," American Journal of Physics, vol. 63, no. 6, pp. 513-520, Jun. 1995.

60. H. G. Schantz, "Electromagnetic energy around hertzian dipoles," IEEE Antennas and Propagation Magazine, vol. 43, no. 2, pp. 50-62, April 2001.

61. S. Mikki and J. Aulin, "The stochastic electromagnetic theory of antenna-antenna cross-correlation in MIMO systems," in 12th European Conference on Antennas and Propagation (EuCAP 2018), April 2018, pp. 1-5.

62. K. A. Lukin, G. P. Pochanin, and S. A. Masalov, "Large-current radiator with avalanche transistor switch," IEEE Transactions on Electromagnetic Compatibility, vol. 39, no. 2, pp. 156-160, May 1997.

63. G. P. Pochanin, "Large current radiator for the short electromagnetic pulses radiation," in UltraWideband Short-Pulse Electromagnetics 4 (IEEE Cat. No.98EX112), June 1998, pp. 149-155.

64. - "Pulse radiation of four-element large current radiator," in IEEE Antennas and Propagation Society International Symposium. 1999 Digest. Held in conjunction with: USNC/URSI National Radio Science Meeting (Cat. No.99CH37010), vol. 4, July 1999, pp. 2722-2725 vol.4.

65. G. P. Pochanin, I. Y. Pochanina, and P. V. Kholod, "Radiation efficiency of the large current 
radiators. electrodynamic simulation," in 4th International Conference on Antenna Theory and Techniques (Cat. No.03EX699), vol. 2, Sep. 2003, pp. 542-545 vol.2.

66. G. P. Pochanin, "Large current radiators," in 2006 3rd International Conference on Ultrawideband and Ultrashort Impulse Signals, Sep. 2006, pp. 77-81.

67. M. Salehi, M. Manteghi, S.-Y. Suh, S. Sajuyigbe, and H. G. Skinner, "A wideband frequency-shift keying modulation technique using transient state of a small antenna," Progress In Electromagnetics Research, vol. 143, pp. 421-445, 2013.

68. M. Salehi and M. Manteghi, "Transient characteristics of small antennas," IEEE Transactions on Antennas and Propagation, vol. 62, no. 5, pp. 2418-2429, May 2014.

69. M. Manteghi, "A wideband electrically small transient-state antenna," IEEE Transactions on Antennas and Propagation, vol. 64, no. 4, pp. 1201-1208, April 2016.

70. — - "Fundamental limits, bandwidth, and information rate of electrically small antennas: Increasing the throughput of an antenna without violating the thermodynamic Q-factor," IEEE Antennas and Propagation Magazine, vol. 61, no. 3, pp. 14-26, June 2019.

71. S. Mikki and A. Kishk, "Electromagnetic scattering by multi-wall carbon nanotubes," Progress In Electromagnetics Research B, vol. 17, pp. 49-67, 2009.

72. K. Cho, Optical response of nanostructures: microscopic nonlocal theory. Berlin New York: Springer, 2003.

73. S. Mikki and A. Kishk, "Quantum particle swarm optimization for electromagnetics," IEEE Transactions on Antennas and Propagation, vol. 54, no. 10, pp. 2764-2775, Oct 2006.

74. S. Mikki and A. A. Kishk, "Infinitesimal dipole model for dielectric resonator antennas using the qpso algorithm," in 2006 IEEE Antennas and Propagation Society International Symposium, 2006, pp. 3285-3288.

75. S. Mikki and A. Kishk, "Theory and applications of infinitesimal dipole models for computational electromagnetics," IEEE Transactions on Antennas and Propagation, vol. 55, no. 5, pp. 1325-1337, May 2007.

76. S. Mikki and Y. Antar, "Near-field analysis of electromagnetic interactions in antenna arrays through equivalent dipole models," IEEE Transactions on Antennas and Propagation, vol. 60, no. 3, pp. 1381-1389, March 2012.

77. S. Mikki and Y. Antar, "A rigorous approach to mutual coupling in general antenna systems through perturbation theory," IEEE Antennas and Wireless Communication Letters, vol. 14, pp. $115-118,2015$.

78. S. Clauzier, S. Mikki, and Y. Antar, "Generalized methodology for antenna design through optimal infinitesimal dipole model," in 2015 International Conference on Electromagnetics in Advanced Applications (ICEAA), Sep. 2015, pp. 1264-1267.

79. — "Design of high-diversity gain MIMO antenna arrays through surface current optimization," in 2015 IEEE International Symposium on Antennas and Propagation USNC/URSI National Radio Science Meeting, July 2015, pp. 9-10.

80. S. Clauzier, S. Mikki, A. Shamim, and Y. Antar, "A new method for the design of slot antenna arrays: Theory and experiment," in 2016 10th European Conference on Antennas and Propagation (EuCAP), April 2016, pp. 1-5.

81. S. Mikki, "Electromagnetic machine learning," in Short course delivered in the 201913 th European Conference on Antennas and Propagation (EuCAP), March, Krakow, Poland, March 2019. 